Published in final edited form as:

J Appl Gerontol. 2020 January ; 39(1): 99-104. doi:10.1177/0733464817750273.

\title{
The role of perceived discrimination and other psychosocial factors in explaining diabetes distress among older African American and White adults
}

\author{
Ishan C. Williams, \\ University of Virginia, School of Nursing, 202 Jeanette Lancaster Way, PO Box 800782, \\ Charlottesville, VA 22903-0782 \\ Olivio J. Clay, \\ University of Alabama at Birmingham, Department of Psychology, 415 Campbell Hall, $17202^{\text {nd }}$ \\ Avenue South, Birmingham, AL 35294-1170 \\ Fernando Ovalle, \\ University of Alabama at Birmingham, Department of Medicine, Division of Endocrinology, \\ Diabetes, \& Metabolism, 702 Faculty Office Tower, 510 20th Street South, Birmingham, AL \\ 35294-3400

\section{Deidra Atkinson,} \\ University of Alabama at Birmingham, Department of Psychology, 438 Stonecrest Drive, \\ Birmingham, Al 35242

\section{Michael Crowe} \\ University of Alabama at Birmingham, Department of Psychology, $15303^{\text {rd }}$ Ave. S., HMB 11, \\ Department of Psychology, Birmingham, AL 35294
}

Ishan C. Williams: icw8t@virginia.edu; Olivio J. Clay: oclay@uab.edu; Fernando Ovalle: fovalle@uab.edu; Deidra Atkinson: dniseatk@uab.edu; Michael Crowe: mgcrowe@uab.edu

\section{Abstract}

Background-Diabetes distress, also referred to as diabetes-related emotional distress, has been shown to contribute to worsening diabetes status over time as well as increased depressive symptoms. Other psychosocial factors, including perceived discrimination, are also related to poorer diabetes outcomes. This study explores the relationships between diabetes distress and several psychosocial variables relevant to health disparities (i.e., race, cognition, social support, physician trust, and perceived discrimination) among older adults with Type 2 diabetes.

Design and Method-Structured telephone interviews were conducted with 148 African American and White adults (age >=65) with T2DM.

Results-Perceived discrimination and physician trust partially explain the relationship between African American race and diabetes distress. Younger age, less satisfaction with social support, and lower physician trust were associated with higher levels of diabetes distress. 
Conclusions-These results highlight the need to address unique stressors, such as perceived discrimination, among racial/ethnic minorities in order to improve diabetes-related outcomes.

\section{Keywords}

type 2 diabetes; physician trust; discrimination; diabetes distress

Type 2 diabetes (T2DM) is a serious worldwide public health challenge, and prevalence has steadily increased over the past three decades (WHO, 2017). However, the burden of diabetes, including disease prevalence and risk of complications, is greater among minorities, particularly African Americans (Anderson et al., 2005). Considering this health disparity, health care providers have an increased challenge to design culturally sensitive and targeted strategies for managing the disease and its complications when assisting minority patients with self-management. Identifying disparities is a first step in understanding what causes them and what can be done to reduce them. As a result, a better understanding of diabetes distress, an assessment of diabetes-related emotional distress (i.e., worries or concerns related to managing a chronic disease), is needed. Diabetes distress has been shown to contribute to worsening diabetes status over time and to increasing depressive symptoms (Carper et al., 2014; Fisher et al., 2008). Individual patients with diabetes may feel as though others do not understand what it means to manage diabetes. Additionally, individuals with diabetes often feel overwhelmed by self-care regimens for managing the disease (Utz, Williams, Jones, \& et al., 2008; Williams et al., 2014).

Managing T2DM, often in conjunction with other chronic conditions, can have a significant impact on one's emotional and physical health, including factors such as anxiety, depression, and other stress (Fisher et al., 2008; Franks, Lucas, Stephens, Rook, \& Gonzalez, 2010). The current investigation utilized the NIA Health Disparities Framework (Hill et al., 2015), which has an emphasis on examining how psychosocial processes can serve as pathways of risk or resilience when examining how an individual's environment can impact their mental and physical health. Previous research suggests that psychosocial factors are related to levels of diabetes distress, which can be attributed to but not limited to, discrimination and poor access to quality health care (LeBron et al., 2014; Wardian \& Sun, 2014). Cognitive health, including problem solving and planning, is an important factor to consider in diabetes management (Primozic, Tavcar, Avbelj, Dernovsek, \& Oblak, 2012). Research also demonstrates that when patients report trust in their physician they perceive greater levels of diabetes self-management and report more capability to meet their diabetes goals (Lafata et al., 2013). In terms of other psychosocial factors, greater satisfaction with support has been demonstrated to be associated with better diabetes-specific quality of life and blood glucose monitoring (Tang et al., 2008). A strong support system is thought to increase resilience of patients with diabetes (Ing et al., 2016; Strizich et al., 2016), allowing them to better manage their diabetes stress. Although these findings highlight psychosocial factors relevant to health disparities in those with diabetes, it is essential for researchers to understand the interrelationship of these factors with levels of distress in older adults with diabetes.

This study primarily aims to examine the association of race, cognition, social support, physician trust, and perceived discrimination with diabetes distress. Better understanding of 
these psychosocial and emotional factors may help facilitate and sustain better selfmanagement of diabetes among older adults. Consequently, this knowledge can ultimately improve the physical health and emotional well-being of older people with diabetes, particularly among African American adults who experience the greatest burden from this health disparity. We hypothesized that: (1) African Americans would report higher levels of diabetes distress than Whites, (2) higher levels of social support across all participants would be associated with less diabetes distress, (3) lower levels of physician trust and higher levels of perceived discrimination would be associated with higher levels of diabetes distress, and (4) physician trust and diabetes distress would each partially explain the effect of race on diabetes distress.

\section{METHODS}

\section{Sample Recruitment and Data Collection}

For the current study, we used data from the University of Alabama at Birmingham (UAB) Diabetes and Aging Study of Health (DASH). The primary aim of DASH was to examine potential disparities between older African Americans and Whites with diabetes among the 252 participants enrolled at baseline (see Jones et al., 2016 and Bowen et al., 2015 for additional study details). Participants included community-dwelling older adults (age 65 and older) from Birmingham, Alabama and surrounding areas as well as patients from a diabetes clinic at UAB. We oversampled African Americans to achieve approximately equal representation compared to Whites.

Potential participants were contacted via mail. If they consented to participate, they received a follow-up telephone call. For the current study, data from the participants remaining at the 2-year follow-up were analyzed. This is when expanded measures of social support, perceived discrimination, physician trust, and diabetes distress were added (IRB\# X090304001). Participants responded to a structured telephone interview including questions about health and demographics, and assessment of cognitive function.

\section{Measures}

Demographic characteristics including age, self-identified race, gender, years of education, and marital status were self-reported. Income was measured in ordinal categories ranging from 1 (less than $\$ 5,000)$ to 9 ( $\$ 100,000$ or greater).

Cognitive functioning was measured using a 13-item modified and validated version of the Telephone Interview for Cognitive Status (TICS-M), which evaluates global cognitive status in older adults (de Jager, Budge, and Clarke, 2003). TICS-M includes domains of orientation, registration/recent memory and delayed recall, attention/calculation, and semantic memory, comprehension and repetition. Scores range from 0 to 39, with a relatively higher proportion of the total score being allocated to the memory component. A score of 20 or lower suggests cognitive impairment.

Two dimensions of social support were measured using questions that have been shown to reliably reflect this factor (Tang et al., 2008). Amount of Social Support was assessed by asking "How much support do you get dealing with your diabetes?" Response options 
ranged from 1 (no support) to 5 (a great deal of support). Satisfaction with Social Support was assessed by participants responding to, "How satisfied are you with the support you get for dealing with your diabetes?" Response options range from 1 (not at all satisfied) to 5 (extremely satisfied). It is notable that this single-item satisfaction question was more highly related to quality of life and glucose monitoring than multiple support measures from the widely used 16-item Diabetes Family Behavior Checklist (Glasgow \& Toobert, 1988).

Perceived discrimination was measured using the Experiences of Discrimination Scale (EOD; Krieger et al., 2005), which first asks the number of settings (up to 9) in which one has experienced discrimination. If perceived discrimination had occurred in any of the 9 settings (yes vs no), questions concerning the frequency of occurrence, and stressfulness of the event(s) are asked. Three scores total were computed using the EOD: number of settings (0-9), a frequency rating (0-45), and a rating of how stressful or upsetting the perceived discrimination was $(0-36)$.

Physician trust was measured using the Trust in Physician Scale (TPS; Anderson \& Dedrick, 1990), an 11-item scale that assesses a participant's belief that his/her doctor's judgments were accurate and the doctor had participant's best interests in mind. Potential scores of TPS range from 11 to 55 with higher scores corresponding to higher levels of physician trust. TPS has demonstrated good internal consistency (Cronbach's alpha $=0.89$ ).

Diabetes Distress, our primary outcome measure, was assessed using the 17-item Diabetes Distress Screening Scale (Polonsky et al., 2005). Participants rate how intensely items caused distress during the past month. Examples include how overwhelmed one feels by the demands of living with diabetes or with managing diabetes routine. Response options for each question ranged from 1 (not a problem) to 6 (very serious problem), with a total score ranging from 17 to 102. The Diabetes Distress Screening Scale has demonstrated excellent internal consistency (Cronbach's alpha $=0.94)$.

Analyses were conducted using SAS V9.3. African Americans and Whites were compared using chi-square for nominal variables and t-tests for continuous measures. Pearson's $r$ was used to assess the relationships between individual variables (demographics, cognition, social support, perceived discrimination, physician trust) and diabetes distress. An initial covariate-adjusted linear regression model included demographic characteristics (age, gender, marital status, education, income, and race), cognitive function, amount of social support, and satisfaction with social support as correlates of diabetes distress. Subsequent models added perceived discrimination and physician trust as potential mediators of the hypothesized race effect.

\section{RESULTS}

The sample consisted of 148 participants (74 Whites, 74 African Americans) with an average age of 72.72 years. In bivariate analyses, when compared to Whites, African American participants were more likely to be female, less likely to be married, had lower education and income, reported higher scores for each measure of perceived discrimination, reported less physician trust, and more diabetes distress (all $\mathrm{p}<.01$ ) (Table 1). Correlational 
analyses revealed that increased age $(\mathrm{r}=-0.183, \mathrm{p}=.0264)$, higher education $(\mathrm{r}=-0.198, \mathrm{p}=$. $0159)$ and income ( $r=-0.290, p=.0004)$, and higher cognitive function $(r=-0.180, p=.0282)$ were each associated with lower diabetes distress. Higher satisfaction with social support ( $\mathrm{r}=$ $-0.421, \mathrm{p}<.0001)$ and higher physician trust $(\mathrm{r}=-0.451, \mathrm{p}<.0001)$ were also associated with lower diabetes distress. Female gender $(r=0.212, \mathrm{p}=.0097)$ and higher perceived discrimination were associated with higher diabetes distress (Pearson's $r$ ranged from 0.197 to 0.240 , p's $<.05$ for associations of the three discrimination measures with diabetes distress).

In a covariate-adjusted model controlling for demographics, social support, and cognition, African Americans still reported higher levels of diabetes distress compared to Whites ( $B=0.171, \mathrm{p}=.0369$; Table 2, Model 1). There were racial differences on perceived discrimination and physician trust, and each was associated with diabetes distress, supporting examination of these variables as mediators of the relationship between race and diabetes distress. Adding each of the 3 perceived discrimination scores individually mediated the racial difference on diabetes distress. Of the perceived discrimination measures, how harmful or upsetting the event was for the participant explained the largest percentage of the association between race and distress (49\%; Table 2; Model 2). Physician trust also served as a mediator of the race effect when added individually, and explained $24 \%$ of the racial difference (Table 2, Model 3). In a final model (Table 2, Model 4), stressfulness associated with perceived discrimination and physician trust together accounted for $60 \%$ of the racial difference in diabetes distress. Significant predictors of higher levels of diabetes distress in the final model included younger age $(B=-0.152, \mathrm{p}=.0389)$, lower satisfaction with social support $(B=-0.321, \mathrm{p}<.0001$, and lower physician trust $(B=-0.274$, $\mathrm{p}=.0003)$.

\section{DISCUSSION}

Consistent with our hypotheses, higher diabetes distress was associated with African American race, higher levels of perceived discrimination, lower levels of physician trust, and lower levels of social support. Racial differences in diabetes distress remained significant after controlling for demographics, social support, and cognition. In addition, physician trust and perceived discrimination accounted for more than half of the association between race and diabetes distress. This is consistent with other research on the impact of stressors such as perceived discrimination on health, and the role of perceived discrimination as a social determinant of health (Williams \& Mohammed, 2009).

Our findings highlight the importance of examining the influence of perceived discrimination on diabetes distress. It is well documented that discrimination is a social determinant of health linked to poor physical and mental health (Healthy People, 2017; Lewis, Cogburn, \& Williams, 2015; Walker et al., 2014; Braveman et al., 2011; Viner et al., 2012). Our results are consistent with a recent study on discrimination and quality of life among people with Type 2 diabetes (Achuko, Walker, Campbell, Dawson, \& Egede, 2016). Achuko and associates found that perceived discrimination was significantly associated with diabetes-related distress, and it served as a pathway influencing the mental health component quality of life score. 
The association of social support and physician trust with diabetes distress in the current investigation suggests that interventions aimed at strengthening social support and physician trust could potentially improve diabetes-related health outcomes within older African Americans and Whites. Efforts should be made to build trust in the patient-provider relationship, enhancing patient outcomes (Bell et al., 2013). Improving health outcomes is also likely to have a significant impact on health resource consumption.

\section{Conclusions}

The current study provides evidence suggesting diabetes distress is related to several psychosocial factors that are linked to health disparities. Limitations of the study include the use of self-report data and the cross-sectional analyses prevent the investigators from specifying causation. Diabetes-related stress is clearly a multifactorial construct, and interventions aimed at reducing it should address diabetes distress directly, as well as the roles of social support, perceived discrimination, and trust in one's physician. Findings highlight the need to address stress factors unique to racial and ethnic minorities, in order to improve diabetes-related outcomes. Our findings support the need to critically examine and measure perceived discrimination as it may be a significant contributor to racial differences among several disease-related conditions (Lewis et al., 2015). Research suggests that the subjective appraisal of perceived discrimination as stressful or upsetting may identify individuals who are at-risk for poor health outcomes (Williams \& Mohammed, 2009). However, attributions of perceived discrimination may need to be obtained over longer time periods to better understand how the exposure of these events influence the onset of physical and mental illnesses (Paradies et al., 2015). Improving physician trust and social support may enhance patient resilience and improve self-management, although further work is needed. Developing interventions that take these critical factors into account, and the intersectionality of these constructs, can potentially lead to better health outcomes along with consumption of fewer resources among African American and White older adults with diabetes.

\section{Acknowledgments}

This work was supported in part by National Institute on Aging (NIA) grant P30AG022838 (UAB Roybal Center) and National Center for Advancing Translational Sciences (NCATS) award number UL1TR00165 (UAB Center for Clinical and Translational Science).

\section{References}

Achuko O, Walker RJ, Campbell JA, Dawson AZ, Egede LE. 2016; Pathways between discrimination and quality of life in patients with type 2 diabetes. Diabetes Technology \& Therapeutics. 18(3):151158. DOI: 10.1089/dia.2015.0305 [PubMed: 26866351]

Anderson RM, Funnell MM, Nwankwo R, Gillard ML, Oh M, Fitzgerald JT. 2005; Evaluating a problem-based empowerment program for african americans with diabetes: Results of a randomized controlled trial. Ethnicity and Disease. 15(4):671. [PubMed: 16259492]

Bell RA, Arcury TA, Ip E, Grzywacz JG, Nguyen H, Kirk JK, Quandt SA. 2013; Correlates of physician trust among rural older adults with diabetes. American Journal of Health Behavior. 37(5): 660-666. DOI: 10.5993/AJHB.37.5.10 [PubMed: 23985289]

Bowen PG, Clay OJ, Lee LT, Vice J, Ovalle F, Crowe M. 2015; Associations of social support and selfefficacy with quality of life in older adults with diabetes. Journal of Gerontological Nursing. 41(12): 21-29. DOI: 10.3928/00989134-20151008-44 
Braveman P, Egerter S, Williams DR. 2011; The social determinants of health: Coming of age. Annual Review of Public Health. 32:381-398.

de Jager CA, Budge MM, Clarke R. 2003; Utility of TICS-M for the assessment of cognitive function in older adults. International Journal of Geriatric Psychiatry. 18(4):318-324. [PubMed: 12673608]

Carper M, Traeger L, Gonzalez J, Wexler D, Psaros C, Safren S. 2014; The differential associations of depression and diabetes distress with quality of life domains in type 2 diabetes. Journal of Behavioral Medicine. 37(3):501-510. DOI: 10.1007/s10865-013-9505-x [PubMed: 23515932]

Fisher L, Skaff M, Mullan J, Arean P, Glasgow R, Masharani U. 2008; A longitudinal study of affective and anxiety disorders, depressive affect and diabetes distress in adults with type 2 diabetes. Diabetic Medicine. 25(9):1096-1101. [PubMed: 19183314]

Franks MM, Lucas T, Stephens MAP, Rook KS, Gonzalez R. 2010; Diabetes distress and depressive symptoms: A dyadic investigation of older patients and their spouses. Family Relations. 59(5):599_ 610. DOI: $10.1111 / \mathrm{j} .1741-3729.2010 .00626 . x$

Glasgow RE, Toobert DJ. 1988; Social environment and regimen adherence among type II diabetic patients. Diabetes Care. 11(5):377-386. [PubMed: 3391088]

Healthy People. Secretary's Advisory Committee on Health Promotion and Disease Prevention Objectives for 2020. HealthyPeople 2020: An Opportunity to Address the Societal Determinants of Health in the United States. www.healthypeople.gov/2020/topics-objectives/topic/socialdeterminants-health (last accessed February 3, 2017)

Hill CV, Perez-Stable E, Anderson NA, Bernard MA. 2015; The National Institute on Aging health disparities research framework. Ethnicity and Disease. 25:245-254. [PubMed: 26675362]

Ing CT, Zhang G, Dillard A, Yoshimura SR, Hughes C, Palakiko D, Kaholokula JK. 2016; Social support groups in the maintenance of glycemic control after community-based intervention. Journal of Diabetes Research. 2016:7913258.doi: 10.1155/2016/7913258 [PubMed: 27563680]

Jones LC, Clay OJ, Ovalle F, Cherrington A, Crowe M. 2016Correlates of depressive symptoms in older adults with diabetes. Journal of Diabetes Research. :8.

Krieger N, Smith K, Naishadham D, Hartman C, Barbeau EM. 2005; Experiences of discrimination: Validity and reliability of a self-report measure for population health research on racism and health. Social Science \& Medicine. 61(7):1576-1596. [PubMed: 16005789]

Lafata JE, Morris HL, Dobie E, Heisler M, Werner RM, Dumenci L. 2013; Patient-reported use of collaborative goal setting and glycemic control among patients with diabetes. Patient Education \& Counseling. 92(1):94-99. DOI: 10.1016/j.pec.2013.01.016 [PubMed: 23433777]

LeBron A, Valerio M, Kieffer E, Sinco B, Rosland A, Hawkins J, Spencer M. 2014; Everyday discrimination, diabetes-related distress, and depressive symptoms among african americans and latinos with diabetes. Journal of Immigrant \& Minority Health. 16(6):1208-1216. DOI: 10.1007/ s10903-013-9843-3 [PubMed: 23689972]

Lewis TT, Cogburn CD, Williams DR. 2015; Self-reported experiences of discrimination and health: scientific advances, ongoing controversies, and emerging issues. Annual Review of Clinical Psychology. 11:407-440. DOI: 10.1146/annurev-clinpsy-032814-112728

Paradies Y, Ben J, Denson N, Elias A, Priest N, Pieterse A, et al. 2015; Racism as a determinant of health: A systematic review and meta-analysis. PLoS ONE. 10(9):e0138511.doi: 10.1371/ journal.pone.0138511 [PubMed: 26398658]

Primozic S, Tavcar R, Avbelj M, Dernovsek MZ, Oblak MR. 2012; Specific cognitive abilities are associated with diabetes self-management behavior among patients with type 2 diabetes. Diabetes Research \& Clinical Practice. 95(1):48-54. DOI: 10.1016/j.diabres.2011.09.004 [PubMed: 21963107]

Polonsky WH, Fisher L, Earles J, Dudl RJ, Lees J, Mullan J, Jackson RA. 2005; Assessing psychosocial distress in diabetes: Development of the diabetes distress scale. Diabetes Care. 28(3): 626-631. DOI: 10.2337/diacare.28.3.626 [PubMed: 15735199]

Strizich G, Kaplan RC, Gonzalez HM, Daviglus ML, Giachello AL, Teng Y, Grober E. 2016; Glycemic control, cognitive function, and family support among middle-aged and older hispanics with diabetes: The hispanic community health Study/Study of latinos. Diabetes Research \& Clinical Practice. 117:64-73. DOI: 10.1016/j.diabres.2016.04.052 [PubMed: 27329024] 
Tang TS, Brown MB, Funnell MM, Anderson RM. 2008; Social support, quality of life, and self-care behaviors amongAfrican americans with type 2 diabetes. The Diabetes Educator. 34(2):266-276. DOI: 10.1177/0145721708315680 [PubMed: 18375776]

Utz SW, Williams IC, Jones R, et al. 2008; Culturally-tailored intervention for rural african americans with type 2 diabetes. The Diabetes Educator. 34(5):854-865. [PubMed: 18832290]

Viner RM, Ozer EM, Denny S, Marmot M, Resnick M, Fatusi A, Currie C. 2012; Adolescence and the social determinants of health. The Lancet. 379(9826):1641-1652.

Walker RJ, Smalls BL, Campbell JA, Williams JLS, Egede LE. 2014; Impact of social determinants of health on outcomes for type 2 diabetes: A systematic review. Endocrine. 47(1):29-48. [PubMed: 24532079]

Wardian J, Sun F. 2014; Factors associated with diabetes-related distress: Implications for diabetes self-management. Social Work in Health Care. 53(4):364-381. DOI: 10.1080/00981389.2014.884038 [PubMed: 24717184]

Williams DR, Mohammed SA. 2009; Discrimination and racial disparities in health: Evidence and needed research. Journal of Behavioral Medicine. 32:20-47. [PubMed: 19030981]

Williams IC, Utz SW, Hinton I, Yan G, Jones R, Reid K. 2014; Enhancing diabetes self-care among rural african americans with diabetes: Results of a two-year culturally tailored intervention. The Diabetes Educator. 40(2):231-239. DOI: 10.1177/0145721713520570 [PubMed: 24478047] 


\section{Table 1}

Bivariate racial differences on variables of interest

\begin{tabular}{|l|l|l|l|l|}
\hline Measures & $\begin{array}{l}\text { African American } \\
\mathbf{n = 7 4}\end{array}$ & $\begin{array}{l}\text { White } \\
\mathbf{n = 7 4}\end{array}$ & $\mathbf{t}\left(\boldsymbol{\chi}^{\mathbf{2}}\right)$ & $\mathbf{p}$-value \\
\hline $\begin{array}{l}\text { Age, mean (SD) } \\
\text { Range: 65-90) }\end{array}$ & $72.12(5.29)$ & $73.32(6.23)$ & -1.27 & .2075 \\
\hline Female gender, n (\%) & $51(68.92)$ & $30(40.54)$ & $(12.03)$ & .0005 \\
\hline Married, n (\%) & $28(37.84)$ & $45(60.81)$ & $(7.81)$ & .0052 \\
\hline Education, mean (SD) & $13.26(2.79)$ & $14.50(2.51)$ & -2.85 & .0051 \\
\hline Income, mean (SD) & $4.35(1.77)$ & $6.00(1.92)$ & -5.44 & $<.0001$ \\
\hline Cognition, mean (SD) & $23.95(4.85)$ & $26.64(4.29)$ & -3.57 & .0005 \\
\hline Amount of support, mean (SD) & $3.42(1.66)$ & $3.82(1.56)$ & -1.53 & .1289 \\
\hline Satisfaction with support, mean (SD) & $4.49(0.90)$ & $4.53(1.00)$ & -0.26 & .7949 \\
\hline Perceived discrimination - settings, mean (SD) & $2.31(2.00)$ & $0.27(0.67)$ & 8.33 & $<.0001$ \\
\hline Perceived discrimination - frequency, mean (SD) & $7.77(8.08)$ & $0.89(2.57)$ & 6.99 & $<.0001$ \\
\hline Perceived discrimination - stressfulness, mean (SD) & $5.73(6.21)$ & $0.46(1.46)$ & 7.10 & $<.0001$ \\
\hline Physician trust, mean (SD) & $43.32(6.86)$ & $46.23(4.99)$ & -2.95 & .0037 \\
\hline Diabetes distress, mean (SD) & $33.11(17.67)$ & $24.55(9.03)$ & 3.71 & .0003 \\
\hline
\end{tabular}

Note: For income: $1=$ less than $\$ 5,000 ; 2=\$ 5000-\$ 11,999 ; 3=\$ 12,000-\$ 15,999 ; 4=\$ 16,000-\$ 24,999 ; 5=\$ 25,000-\$ 34,999 ; 6=\$ 35,000$ $\$ 49,999 ; 7=\$ 50,000-\$ 74,999 ; 8=\$ 75,000-\$ 99,999$; and $9=\$ 100,000$ or greater. 
Table 2

Covariate-adjusted associations of variables of interest and diabetes distress.

\begin{tabular}{|l|l|l|l|l|}
\hline Measures & Model 1 & Model 2 & Model 3 & Model 4 \\
\hline Age & -0.138 & -0.138 & $-0.152^{*}$ & $-0.152^{*}$ \\
\hline Female gender & 0.142 & 0.124 & 0.122 & 0.109 \\
\hline Married & 0.104 & 0.090 & 0.106 & 0.095 \\
\hline Education & -0.007 & -0.006 & -0.016 & -0.015 \\
\hline Income & -0.152 & -0.168 & -0.115 & -0.129 \\
\hline Cognition & -0.085 & -0.092 & -0.052 & -0.058 \\
\hline Amount of support & 0.046 & 0.040 & 0.034 & 0.030 \\
\hline Satisfaction with support & $-0.400^{* * *}$ & $-0.402^{* * *}$ & $-0.315^{* * *}$ & $-0.321^{* * *}$ \\
\hline African American Race & $0.171^{*}$ & 0.088 & 0.131 & 0.069 \\
\hline Perceived discrimination - stressfulness & & 0.151 & & 0.114 \\
\hline Physician trust & & & $-0.288^{* * *}$ & $-0.274^{* *}$ \\
\hline
\end{tabular}

Note: Standardized betas $(B)$ are presented in the table.

**** $\mathrm{p}<.0001$,

* $\mathrm{p}<.01$,

p $<.05$ 